\title{
Die Bluteosinophilie als Indikator für die jeweilige Reaktionsfähigkeit des Organismus.
}

\author{
Von \\ Prof. Otfried Müller, und Prof. Otto Brösamlen. \\ Vorstand der Klinik. Oberarzt dor Klinik.
}

(Eingegangen am 3.10.1921.)

\begin{abstract}
Wenn man vor etwa 10 Jahren eine Reihe erfahrenster Tuberkuloseärzte über die Auswahl der Fälle befragte, welche sich zur Tuberkulintherapie eigneten, so bekam man - soweit die Herren überhaupt zum Tuberkulin raten konnten keine präzis formulierte Auskunft. In der üblichen Weise wurden die leichten Fälle - abgesehen von bestimmten Ausnahmen - als geeignet, die schweren als ungeeignet bezeichnet. Für die große Zahl der mittleren Fälle hieß es, daß die Tuberkulinkur eine Sache ausgiebiger persönlicher Erfahrung sei, und daß man in der Regel nachher wisse, ob man wohl getan habe, sie in die Wege zu leiten.

Bei dieser Lage der Dinge erschien es dringend erwünscht, einen objektiven Leitfaden zu gewinnen, der es ermöglichen möchte, auch ohne die Summe lebenslänglicher, subjektiver Erfahrungen möglichst frühzeitig zu erkennen, wann es an der Zeit sei, cine eingeleitete Tuberkulinkur vorsichtiger oder kräftiger zu dosieren, oder sie ganz abzubrechen. Da nun die Erfahrung vorlag, daß3 schwere infektiös-toxische Belastungen des Organismus, wie z. B. bei Typhus auf der Höhe der Krankheit und Sepsis in vorgeschrittenen Stadien die Blutcosinophilie zu starker Verringerung resp. völligem Schwinden bringen, so lag es nahe, cliese Verhältnisse auch unter der Tuberkulineinwirkung eingehender zu studieren. O. Müller veranlaßte deshalb Brösamlen (1), der einige Zeit bei Nägeli geweson war, sich auf der Tuberkuloseabteilung der Tüibinger Klinik näher mit dieser Frage zu beschäftigen.

Bekannt waren zu jener Zeit nur die Untersuchungen von Sahli und Fau connet (2), die im Anschluß an Tuberkulininjektionen eine Vermehrung der Gesamtzahl der weißen Blutkörperchen eintreten sahen. Über die Eosinophilen fanden sich einige Zufallsangaben. So sahen z. B. Bisch of und Grawitz (3) in zwei Fillen nach Tuberkulininjektionen eine ganz enorme Vermehrung der eosinophilen Zellen auftreten, die in einem Falle noch 2 Monate nach der Injektion nachweisbar war. Und Botkin (4) fand in 3 von 4 untersuchten Fiillen


eine verstïrkte Eosinophilie nach Tuberkulindarreichung. Fauconnet (2) bestritt diese Angaben ganz entschieden.

Brösamlen (1) ging deshalb systematisch an einem größeren Material vor, untersuchte morgens um 9, mittags um 12 und abends um $6 \mathrm{Uhr}$, verwendete zunächst Fälle, die zur probatorischen, diagnostischen Injektion (beginnend mit $2 / x_{0}$, steigend über 1 allmählich auf $5 \mathrm{mg}$ Alttuberkulin in 48 stündigen $\mathrm{Ab}$ ständen) kamen, machte zwei Tage Vorperiode und kontrollierte dann den ganzen Verlauf bis zur Rückkehr des Blutbildes zur Norm. Er kam bei diesen Untersuchungen zu folgenden Schlüssen:

1. Bei klinisch Gesunden wird das Blutbild durch die Tuberkulininjektionen nicht wesentlich beeinflußt.

2. Bei Tuberkulösen sieht man neben einer akuten Leukozytose eine durch die Injektion bedingte Eosinophilie des Blutes auftreten. Diese Eosinophilie ist in ihrer Bedeutung prinzipiell zu scheiden von der postinfelstiösen, also sekundären Eosinophilic, wie man sie in der Rekonvaleszenz fast aller Infektionskrankhciten sieht.

3. Die Eosinophilie ist in klinischer Dignität gleichzusetzen den Erscheinungen, wie sie als Allgemein- und Fieberreaktion bekannt sind. Bezüglich des Herdes sagt sie natiurlich nichts. Die Reaktion ist feiner, als die klinisch manifest werdenden Erscheinungen, da sie ihnen häufig vorauseilt.

4. Die Eosinophilie bei Tuberkulininjektionen ist als Folge eines anaphylaktischen Zustandes aufzufassen und entspricht einer Schutzreaktion des Organismus.

Die Eosinophilie blicb dann aus oder war wenigstens nicht so hochgradig, wenn der betreffende Organismus noch unter dem Einfluß einer vorausgegangenen Injektion stand, also hohe Leukozytenzahl und hohe Eosinophilenwerte aufwies. In solchen Fällen trat die Eosinophilie erst mit dem Abklingen der Temperatur auf. War aber der Zwischenraum zwischen zwei aufeinander folgenden Injektionen groß und hatte der Organismus genügend Zeit; sich zu erholen, dann war auch das Ansteigen der Eosinophilen nach der Injektion wieder zu beobachten.

Erwähnenswert ist weiter, daß nach den Befunden Brösamlens (1) an der Zunahme der Gesamtzahl der weißen Blutkörper nach den Injektionen vorzugsweise die neutrophilen Leukozyten beteiligt waren. In einem Falle wurde im Anschluß an jede Injektion das Auftreten einer primären Lymphozytose beobachtet. Sonst trat eine Lymphozytose nur dann ein, wenn eine länger oder kürzer dauernde Neutrophilie vorausgegangen war.

Nachdem die Veränderungen des Blutbildes bei probatorischen Reizdosen von Tuberkulin klargelegt waren, ging Brösamlen (5) dazu über, auch bei mittelschweren, leicht fiebernden Kranken zu untersuchen, die mit den üblichen sehr kleinen Dosen von Neutuberkulin-Bazillenemulsion gespritzt wurden. Die Untersuchungen wurden in der oben angegebenen Häufigkeit ausgeführt. Es zeigte sich, daß Kranke, welche die Injektionen gut vertrugen, und deren $\mathrm{Zu}$ stand sich unter der Tuberkulintherapie subjektiv und objektiv besserte, regelmäßig mit einer Vermehrung der eosinophilen Zellen reagierten. Die Eosino. philie blieb aber aus, sowie Störungen im Verzug waren, sei es nun, da $\mathbf{s}$ sie durch die Injektionen als solche, oder durch interkurrente Erkrankungen verursacht 
waren. Interessanterweise konnte man durch die Kontrolle des Blutbildes die Störung voraussagen, ehe klinische Anhaltspunkte hierfür vorlagen. So wurden die Untersucher z. B. in einem Falle durch das Ausbleiben der sonst regelmäßig aach den Injektionen auftretenden Eosinophilie darauf aufmerksam gemacht, daß etwas nicht in Ordnung sein müsse. Kurz darauf zeigte sich eine Angina. Genau so lagen die Verhältnisse bei solchen Kranken, die von einem gewissen Zeitpunkte $a b$ die Injektionen nicht mehr vertrugen. Schon reichlich lange, bevor klinisch nachweisbare Reaktionen ausgelöst wurden, machte sich die Störung durch das Ausbleiben der Eosinophilie geltend, so daß man frühzeitig genug durch Reduktion der Dosis und durch Verlängerung der Pausen der drohenden Gefahr begegnen konnte.

Weiter fanden sich bei diesen Untersuchungen Fälle, die schon von den ersten Injektionen ab und auch im weiteren späteren Verlauf nie eine Vermehrung der Eosinophilen aufwiesen, im Gegenteil eher eine Verminderụng dieser Zellen im Blute beobachten ließen. Es handelte sich dabei um Kranke, die entweder bald gröbere Reaktionen zeigten, oder aber um solche, die oberflächlich betrachtet, die Injektionen ganz gut, weil reaktionslos, vertrugen, deren Zustand aber bezüglich des objektiven Befundes unter der Tuberkulintherapie sichtlich schlechter wurde.

Dementsprechend empfahl unsere Klinik bei der Durchführung einer Tuberkulinkur in zweifelhaften Fällen am Tage vor der Injektion, am Tage der Injektion und am Tage nach derselben je morgens um 9, mittags um 12 und abends um 9 Uhr Blut zum Ausstrich zu entnehmen und zu differenzieren.

Später wurde die Technik nach den Angaben Dungers (16) einfacher und weniger zeitraubend. Man brauchte nicht mehr zu differenzieren, sondern konnte sich begnügen, die Eosinophilen nach entsprechender Färbung in der Zählkammer in absoluter Zahl auszuzählen.

Die Angaben Brösamlens wurden von Schenitzki (6) im Jahre 1917 nachgeprüft und in wesentlichen Punkten bestätigt. Schenitzki schreibt: „Die Lungentuherkulose zeigt im initialen Stadium meistens ein normales Blutbild mit fast stets reduzierten Hämoglobinwerten und Erythrozytenzahlen, oft finden wir auch mehr oder weniger deutliche Lymphozytosen. Bei fiebernden Lungentuberkulosen finden wir ziemlich oft Neutrophilie, die Übergangsformen und Monozyten sind meistens vermehrt; die Eosinophilen normal oder häufiger vermindert. Nach Tuberkulininjektionen tritt meistens schon nach kleinen therapeutischen Dosen Neutrophilie als Ausdruck der begleitenden reaktiven Erscheinungen ein; sie muB aber nicht immer vorhanden sein, und kann sich auch ohne sichtbare reaktive Erscheinungen zeigen. Nach Wiederholung derselben Dosis kann die Neutrophilie trotz der reaktiven Erscheinungen ausbleiben, ja es kann sogar die Zahl der Neutrophilen zurückgehen. Auch Lymphozytose oder Eosinophilie kann nach Tuberkulininjektionen eintreten. Eosinophilie können wir (mit Brösamlen) immer als Zeichen der Besserung auffassen. Das Fehlen derselben ist aber entgegen Brösamlen, nicht an und für sich ein Zeichen der Verschlimmerung oder schlechter Bedeutung."

Auf diesen letzteren Einwand hat Brösamlen (7) erwidert: ,Schenitzki hat mich mißverstanden, wenn er aus meinen Ausführungen die Ansicht herausliest, als ob Kranke, welche durch die Tuberkulinbehandlung gebessert werden, 
unbedingt nach jeder Injektion mit einer Vermehrung der eosinophilen Leukozyten reagieren müßten. Das kann natürlich der Fall sein, bildet aber durchaus nicht die Regel. Ich bin nach wie vor der Meinung, daß das Ausbleiben der Eosinophilie nach einer Injektion als ungünstiges Zeichen aufzufassen ist, insofern, als dadurch zum Ausdruck kommt, daß die Dosierung dem Reaktionszustand des Körpers nicht angepaßt war. Bei der Feinheit der Reaktion braucht es deshalb nicht sogleich zu einer Verschlimmerung des Krankheitsprozesses zu kommen. Geht man aber ohne Rücksicht darauf mit der Dosis höher, so wird es früher oder später auch dazu kommen können. Der Hauptwert der Blutuntersuchung liegt gerade darin, daß man rechtzeitig gewarnt wird und durch Verringerung der Dosis und durch das Einschieben größerer Pausen zwischen den Injektionen die drohende Gefahr abwenden kann."

Wir bringen diese kleine Kontroverse hier ausführlicher, weil sie einen prinzipiellen Punkt berührt, der leicht zu Mißverständnissen führen kann. Nach Ehrlich werden die eosinophilen Zellen durch bestimmte chemische Reize aus dem Knochenmark in die Blutbahn ausgeschwemmt. Letzten Endes stellt also die Eosinophilenkurve eine Funktionskurve des Knochenmarkes dar. Die ausgeschwemmten Zellen betätigen sich dann im Organismus als Verteidigungsmittel gegenüber allen möglichen Toxinen, die eine Gefahr für sein Wohlbefinden werden können. Schlecht (9 und 10) hat den Vorgang der toxisch bedingten reaktiven Eosinophilie als anaphylaktisch angesprochen, weil er bei Mensch und Tier im anaphylaktischen Schock eine erhebliche Eosinophilie auftreten sah.

Wie dem nun auch sei, eine toxisch auszulösende Eosinophilie wird stets eine Relation zwischen der momentan vorliegenden Reizmenge und der ebenfalls momentan gegebenen Reaktionsfähigkeit des Knochenmarkes darstellen. Beide Faktoren sind in der Zeit stark variabel. Man überlege das an folgendem Beispiel: Ein Tuberkulöser bekommt heute bei Bettruhe ein Hunderttausendstel Milligramm Alttuberkulin und reagiert darauf mit Steigen der Eosinophilen. In drei Tagen bekommt er die gleiche Dosis nach einem Spaziergang und reagiert darauf mit Sinken. Während des Spazierganges hat er Autotuberkulin gebildet, was während der Bettruhe nicht geschah. Dieses addiert sich in seiner Reizwirkung auf das Knochenmark zu der Einspritzung. Die Reaktionsfähigkeit des Kranken ist infolge seiner Krankheit bereits eine beschränkte. Demgemäß wird er durch die Summationswirkung überdosiert. Daraus folgt erstens, daß man immer unter gleichen Verhältnissen, $d . h$. bei Bettruhe und um die gleiche Tageszeit spritzen soll, und daß zweitens eine Schwalbe noch keinen Frühling macht, sondern nur auf die momentane Lage hinweist.

Man wird also durch den ersten Versager bei der Eosinophilenprüfung nur darauf hingewiesen, daß zur Zeit ein Mißverhältnis zwischen Reizwirkung und Reaktionskraft besteht. Ob das durch eine danernde oder nur durch eine rorübergehende, vielleicht durchaus nebensächliche,Ursache\{beginnender Schnupfen, leichte Indigestion) bedingt ist, erfährt man nicht. Darum wird man nach einer angemessenen Zeit wieder versuchen, entweder mit der gleichen oder mit einer kleineren Dosis. Kommt es wieder zu keiner Vermehrung der Eosinophilen, so wird man länger zuwarten und vorsichtig tastend weiter probieren. Bleibt auch dann die Antwort eine negative, so sollte man nach unseren recht ausgedehnten Erfahrungen nicht nur bei der Tuberkulinwirkung nieht zuwarten, 
bis der Körper seine Realitionsunfähigkeit durch ernstliche Verschlechterung les Befundes und des Befindens ad oculos demonstriert. Also nochmals: Die Eosinophilenprüfung ist eine Stichprobe, die nur für den Moment Gültigkeit hat, in dem sie vorgenommen wurde. Fallen mehrere kritisch und vorsichtig ausgeführte Stichproben in negativem Sinne aus, so ist man zu Jer Annahme berechtigt, daß die Reaktionsfähigkeit des zu prüfenden Organismus nicht durch eine zufällige und vorübergehende Komplikation beeinträchtigt ist, sondern daß die Krankheit den Körper für lüngere Zeit, vielleicht für dauernd reaktionsunfähig gemacht hat. Das Wort dauernd ist natürlich auch sehr mit Vorsicht zu gebrauchen, denn ein ernstlicher tuberkulöser Prozeß, der im ganzen Jahre 1921 reaktionsunfähig ist, kann ja schließlich bei geeignetem Verhalten im Jahre 1922 so weit gebessert sein, daß er wieder reaktionsfähig wird.

Diese Gedanken finden in manchen Ausführungen der letzten Zeit ihre Unterstützung. So schreibt z. B. Ha yek (I0): , Wir können die Eigenart unserer Tuberkulose zusammenfassen:

Langsame Entwicklung; oft jahre- und jahrzehntelang im Sinne der praktischen Heilkunde erscheinungslos.

Wechselvoller Verlauf.

Diese Erkenntnis führt zu dem Gebot, die Wechselwirkung zwischen 'Tuberkelbazillus und Menschenkörper möglichst frühzeitig zu erfassen. Das ist unleugbar schwierig, für unsere Tätigkeit unhequem, aber die Wichtigkeit dieses Gebotes für unsere Vorbeugungs- und Heilbestrebungen ist heute klar erkannt. Seine Erfüllung ist das Arbeitsziel der Zukunft.“ Und an anderer Stelle: „Für den Eingeweihten steht heute die zielsichere Linio fest: Erfassung der immunbiologischen Kräfteverhältnisse und ihrer gesetzmäßigen Veränderungen: Erforschung von Methoden, um dieses Kräfteverhältnis zugunsten des Körpers zu ändern."

Weiter heißt es in einem Aufsatze über Schwellenreiztherapic von Zimmer (11): ,Die für die ganze durch Reizwirkung auf Leistungssteigerung hinzielende Therapie geradezu brennende Frage ist die Dosierung des Reizes. Hier ergeben sich eine ganze Anzahl von Tatsachen, die zum Verständnis dieser Frage notwendig sind aus folgenden Überlegungen: Auf jeden Reiz muß eine Reaktion erfolgen. Wo nichts reagiert, kann nicht gereizt werden. Die Reaktion ist die Antwort des Gewebes auf einen Reiz, der sich darin offenbart, daß sich dic diesem Gewebe eigentümlichen Funktionen in ihrer Stärke ändern. Die Reaktion kann sich in Form einor Leistungssteigerung geltend machen. Doch ist die Leistungssteigerung (Anregung) der Zellen nicht unbegrenzt, wir müssen einmal zu einem Optimum der Leistungsfähigkeit der Zelle kommen und damit diejenige Schwelle erreichen, bis zu der der Reiz noch eine dauernde Leistungssteigerung hervorbringt. Bei Reizungen, die über diesen Schwellenreiz hinausgehen, nimmt die Leistung der Zelle allmählich wieder ab, bis dadurch eine Leistungsverminderung und schließlich eine Lähmung hervorgerufen wird. Wir können also durch Abstufung des Reizes gegenteilige Wirkungen im Körper erzielen.

In zustimmendem Sinne werden neuerdings die Untersuchungen Brösam lens bezüglich der Tuberkulinwirkung auf die Eosinophilenkurve von Fränkel (12) und Blumenfeld (13) referiert; am eingehendsten befaßt sich auf Grund ausgiebiger Nachuntersuchungen letzthin Rom berg (14) mit der Sache. Er schreibt: 
„Recht nützlich ist die genaue Kontrolle des weißen Blutbildes bei der diagnostischen und therapeutischen Tuberkulinanwendung. Schon Sahli betonte das Auftreten von Leukozytose nach Tuberkulineinspritzungen, die noch keine andere Reaktion erkennen lassen. Nach unserer wie anderer Autoren Erfahrung ist das freilich nicht regelmäßig. Brösamlen hob, wie schon erwähnt; die Eosinophilen als frühes Kennzeichen der Reaktion bei prognostisch günstigen Fällen hervor. Auch das ist völlig zutreffend, wenngleich, wie wir sofort sehen werden, mit einer gewissen Einschränkung." Diese Ausnahme besteht in der paradoxen Reaktion einer Tuberkulineinspritzung bei einem Bronchialasthma. Es ist klar, daß bei bereits bestehenden Eosinophilien, wie anläßlich von Asthma oder Helminthiasis eine gewisse Vorsicht in der Beurteilung am Platze ist. Im übrigen will Romberg schon aus der bei diagnostisch-probatorischen Tuberkulininjektionen auftretenden Eosinophilie prognostische Schlüsse herleiten.

Man kann somit heute wohl sagen, unsere Empfehlung der Eosinophilenkurve zur Beurteilung der Tuberkulinwirkung hat sich durchgesetzt. Darüber, daß ein Steigen der Eosinophilen nach Tuberkulinanwendung ein gutes Zeichen ist, herrscht, soviel wir aus der Literatur sehen, überhaupt absolute Einigkeit. Daß man beim Sinken der Kurve bezüglich gewisser Komplikationen (Asthma, tierische Parasiten) Kritik anzuwenden hat, versteht sich nebenher. Daß dieses Sinken aber in unkomplizierten Fällen prognostisch ungünstig anzusehen ist, wird sich ebenso, wie es von den letzten Untersuchern (Romberg) bereits anerkannt ist, auch sonst durchsetzen, wenn man, wie oben ausgeführt, nicht an einzelnen Stichproben hängen bleibt, die naturgemäß ein durch momentane Einflüsse entstelltes Bild geben können.

Nachdem das Verhalten der Eosinophilenkurve bei diagnostischen und therapeutischen Tuberkulininjektionen hinreichend geklärt war, ging Brösamlen (17) dazu über, die entsprechende Wirksamkeit verschiedener Vakzinen zu studieren. Die Untersuchungen wurden unter der Einwirkung einer großen Zahl Autovakzinen von Koli, Strepto- resp. Staphylokokken und bei Arthigon vorgenommen. Bei der Durchführung der Vakzinetherapie wurde nach dem Grundsatz verfahren, gröbere Reaktionen nach Möglichkeit zu vermeiden. Dementsprechend wurde mit kleinen Keimzahlen begonnen. Mit den Blutuntersuchungen wurde einige Tage vor Ausführung der ersten Injektion angefangen. Das Blut wurde zweimal täglich (morgens um 9 und nachmittags um $6 \mathrm{Uhr}$ ) untersucht.

Es zeigte sich, daß der an einer Koliinfektion erkrankte und spezifisch behandelte Patient genau diesclbe Blutreaktion aufwies, wie der mit Staphylokokkenvakzine beeinflußte Aknekranke oder der mit Arthigon gespritzte Gonorrhoiker, oder der mit Bazillenemulsion behandelte Tuberkulöse. „Es bestehen also in dem Verhalten des Blutes im Anschluß an die Vakzineinjektionen bei den verschiedenen chronischen Infektionskrankheiten keine prinzipiellen Unterschiede. Die Veränderungen sind in der Regel recht charakteristischer Art. Nach jeder Injektion kommt es mit ziemlicher Regelmäßigkeit zu einer mehr oder minder beträchtlichen Vermehrung der weißen Blutkörperchen. Die Vermehrung setzt schon wenige Stunden nach der Injektion ein und ist auch vorhanden, wenn klinisch nachweisbare Reaktionserscheinungen vollkommen fehlen. Nur in einzelnen Fällen sinkt die Zahl der weißen Blutkörperchen un- 
mittelbar nach der Injektion zunächst unter die Norm und steigt erst dann zu höheren Werten an. Die Reaktion ist im allgemeinen nach 2-3 Tagen abgeklungen. An der Vermehrung der weißen Blutkörper nehmen in erster Linie die neutrophilen Leukozyten teil. Bei unkomplizierten Fällen werden aber auch die eosinophilen Zellen in vermehrter Menge in die Blutbahn ausgeschwemmt. Es handelt sich dabei um eine primäre Eosinophilie, die im unmittelbaren Anschluß an die Injektion entsteht und gleichzeitig mit der Neutrophilie in Erscheinung tritt. Scharf zu trennen davon ist die Eosinophilie, welche nicht selten im Verein mit einer Lymphozytose, aber auch ohne eine solche nach dem Abkzlingen der Reaktion zu beobachten ist. Diese Erscheinung muß als posttoxischer oder postinfektiöser Vorgang gedeutet werden und entspricht der Eosinophilie, welche wir in der Rekonvaleszenz bei gewissen Infektionskrankheiten (Typhus, Pneumonie) zu sehen gewohnt sind."

Auch hier ist naturgemäß die Deutung der Veränderungen des weißen Blutbildes mit viel Kritik und nicht grobschematisch vorzunehmen, denn ,ganz so gesetzmäßig, wie man nach den bisherigen Ausführungen annehmen könnte, tritt die Eosinophilie nicht auf. Wir haben schon früher auf das Ausbleiben der Reaktion bei Gesunden hingewiesen und haben dieselbe Beobachtung bisweilen auch bei Kranken gemacht. Die Ursachen für den negativen Ausfall können verschiedener Natur sein. Die Art der Dosierung und die jeweilige Reaktionsfähigkeit des betreffenden Kranken spielen dabei eine entscheidende Rolle. Auch die Beschaffenheit des Präparates ist für den Ausfall der Reaktion nicht gleichgültig. Schon geringe Bakterienmengen sind imstande, cine Vermehrung der eosinophilen Zellen im Blut hervorzurufen. Mittelgroße Dosen bewirken im allgemeinen genau dasselbe. Sehr große Dosen haben dagegen nicht nur keine Vermehrung, sondern im Gegenteil eine Verminderung der eosinophilen Zellen zur Folge.

Diese Beobachtungen stehen in vollem Einklang mit den Angaben von Wright, welcher nach der Einverleibung kleiner Bakterienmengen die Opsoninkurve steigen sah, während sehr große Mengen die antibakterielle Kraft des Blutes herabsetzten. Die Begriffe groß und klein sind natürlich sehr dehnbar und individuell verschieden zu bewerten. Es kann z. B. ein und dieselbe Bakteriendosis bei einem Patienten prompt die Eosinophilie auslösen und bei einem andern nicht.

Damit kommen wir zum andern Punkt, nämlich zu dem Einflußdor Reaktionsfähigkeit des Organismus auf das Zustandekommen der Eosinophilie. Ist die Reaktionsfähigkeit des Körpers gut, so wird man auf die Injektion einer nicht allzu großen Bakterienmenge einen positiven Ausfall der Reaktion erwarten dürfen. Liegt sie aus irgendwelchem Grunde danieder, so muß man mit dem Ausbleiben der Eosinophilie rechnen. So vermißt man z. B. die Eosinophilie immer dann, wenn der betreffende Patient noch unter dem Eindruck einer vorausgegangenen Injektion steht und hohe Eosinophilenwerte aufweist. Wird zu diesem Zeitpunkt eine neue Injektion vorgenommen, so sinkt die Zahl der cosinophilen Zellen unter Umständen sogar auf unternormale Werte.

Einen weiteren Beweis für die Abhängigkeit der Eosinophilie von dem Reaktionszustand des Körpers bildet dic Beobachtung, daß interkurrente, selbst ganz leicht verlaufende Krankheiten das Auftreten der Eosinophilie nach der 
Injektion hindern bei Dosen, die vorher eine solche zur Folge hatten. Daran vermag auch eine wesentliche Verringerung der einverleibten Bakterienmenge nichts zu ändern. Sie tritt vielmehr erst dann wieder in Erscheinung, wenn bei erneuter Injektion die dazwischen getretene Krankheit abgeklungen ist."

Wir haben also hier genau die gleichen Verhältnisse wie beim Tuberkulin. Die eintretende Eosinophilie ist nach Schlecht (8 und 9) der ,Ausdruck einer Reaktion des Körpers gegen die durch die Zufuhr artfremden Eiweißes sich bildenden toxischen Abbauprodukte". Diese Reaktion hat nach ihm die Bedeutung eines ,schützenden Vorganges" und er schreibt ihr demgemäß eine ,günstige Bedeutung“" zu. Der schützende Vorgang wird nur ausgelöst, wenn toxische Belastung und Reaktionsfähigkeit des Körpers in einem optimalen Verhältnis zueinander stehen. Ist das vorübergehend nicht der Fall, so empfiehlt sich das zeitweise Aussetzen der Behandlung. Ist das dauernd nicht der Fall, so hat man die Behandlung ganz zu sistieren. Da das optimale Verhältnis aus den geschilderten Gründen leicht einmal gestört sein kann, so empfiehlt es sich, aus einzelnen Versagern keine allzu weit gehenden Schlüsse zu ziehen. Auf der andern Seite wird es natürlich auch nicht empfehlenswert sein, in allzu optimistischer Hoffnung auf eine vorübergehende Indisposition weiter zu machen, bis eine schwere klinische Reaktion da ist.

Wir haben im Laufe der Jahre eine sehr große Anzahl von Vakzinebehandlungen aller Art unter Eosinophilenkontrolle durchgeführt (die von Brösamlen in seiner Publikation angegebenen Zahlen sind längst um ein Vielfaches gestiegen). Wir können sagen, daß wir sehr zufrieden waren. Wir haben herausgebracht, daß ein Präparat nichts taugte, weil jede Eosinophilie dauernd ausblieb, ohne daß der Zustand des Kranken das erklärlich erscheinen ließ. Wir haben erfahren, ob wir rascher oder langsamer vorgehen durften und vor allem, wann es Zeit zum Aufhören war. Unsere Kranken hatten entschieden den Vorteil davon.

Neuestens erleben wir, daß man das weiße Blutbild auch zur Erforschung anderer reiztherapeutischer Wirkungen verwendet. So schreibt letzthin Rolly (15): „Genau so wie es Schlecht bei Seruminjektionen nachgewiesen hatte, findet sich auch nach den Milchinjektionen usw. eine manchmal beträchtliche Leukozytose bis zu 30000 und mehr, welche, je nach dem vorliegenden Krankheitsfall und der durch die Injektion gesetzten Dauer und dem Grade der örtlichen Reaktion verschieden lange Zeit anhalten kann. Die neutrophilen und eosinophilen Leukozyten sind relativ und absolut an Zahl gesteigert, während gleichzeitig die Lymphozyten relativ abnehmen und erst in der Rekonvaleszenz nach dem akuten Stadium wieder steigen, um dann, wie mein Assistent Weicksel zeigen wird, bei solchen Fällen, bei welchen die Einspritzung von günstiger Wirkung begleitet ist, auf höhere Werte vor der Einspritzung in die Höhe zu gehen."

Nachdem allseitig anerkannt war, daß dem Steigen der Eosinophilenkurve nach Einverleibung artfremden Eiweißes eine prognostisch günstige Bedeutung zukommt, haben wir an der Tübinger Klinik auch Untersuchungen darüber veranlaßt, ob der Einfluß physikalischer Heilmittel beim Tuberkulösen ebenfalls zu einer Veränderung des weißen Blutbildes führt. Wir gehen dabei zunächst von der Anschauung aus, daß durch Sonnen-, Höhensonnen- und Röntgen- 
bestrahlungen des Tuberkulösen Organismus Autotuberkulin in die Blutbahn gebracht werden kann, und daß dieses imstande sein dürfte, die anaphylaktische Knochenmarkreaktion hervorzurufen. Wir setzten diese Anschauung, die wir einstweilen nur hypothetisch aussprechen, in Analogie mit den Temperatursteigerungen, wie sie sich nach kräftigeren Bestrahlungen in der bekannten Weise finden.

Aus einer großen Anzahl von Fällen, die wir diesbezüglich haben durchuntersuchen lassen, wählen wir dic folgenden aus:

\section{Natürliche Sonnenbestrahlung:}

1. A. Sch., 27 Jahre alt, doppelseitige kavernöse Lungentuberkulose beider Oberlappen mit starker Neigung zur Bindegewebsbildung, Stadium III. Pleuraschwarte links, subfebrile Temperaturen, Bazillenbefunde positiv, Ernährungszustand gut.

Absolute Eosinophilenzahlen

am 15. VII. 8 Uhr vormittags 189,12 Uhr mittags 204,6 Uhr abends 177 ;

am 16. VII. 8 Uhr vormittags 180.

Von 9-9 $1 / 4$ Sonnenbestrahlung ohne Eintritt deutlicher Hautrötung

12 Uhr mittags 362,3 Uhr nachmittags 241, 6 Uhr abends 275;

am 17. VII. $8 \mathrm{Uhr}$ vormittags 216 .

Hier findet sich also eine beträchtliche Vermehrung der Eosinophilentahl nach der Sonnenbestrahlung (mittags 12 Uhr $182 \mathrm{mehr}$ als an gleichen Tag morgens um 8). Die Patientin wurde nach dreimonatlicher Kurdauer in nahezu unverändertem Zustande entlassen. Eine Verschlechterung des Lungenbefundes hatte nicht stattgefunden, das Körpergewicht hatte um $1 \mathrm{~kg}$ zugenommen. Temperaturen noch subfebril. Sie war also in einem ziemlich stabilen Zustande und ertrug demgemäß die Sonnenbestrahlung besser, als man sonst bei Kranken mit so ausgedehnten, namentlich so stark destruktiven Prozessen zu sehen gewohnt ist.

2. A. H., 34 Jahre alt, doppelseitige Lungentuberkulosc, mehr schrumpfend als zerfallend. Stadium III. Beginnende Larynxtuberkulose, subfebrile Temperaturen, Bazillenbefund positiv, schlechter Ernährungszustand.

Absolute Eosinophilenzahlen

am 29. VI. 8 Uhr vormittags 142,12 Uhr mittags 154,6 Uhr abends 165 ;

am 30. VI. 8 Uhr vormittags 158.

Von 8.10 Uhr bis 8.25 Uhr Sonnenbestrahlung ohne Eintritt deutlicher Hautrötung. 11 Ulhr vormittags 297,2 Uhr nachmittags 223 , 5 Uhr nachmittags 248; am 1. VII. 8 Uhr vormittags $220,6 \mathrm{Uhr}$ abends 197 ;

am 2. VII, \& Uhr vormittags 223 .

Von 8.20 Uhr bis 8.45 Uhr Sonnenbestrahlung mit Eintritt leichter Hautrötung. $11 \mathrm{Uhr}$ vormittags $272,3 \mathrm{Uhr}$ nachmittags $381,6 \mathrm{Uhr}$ abends 316.

Hier findet sich also eine mäßige Vermehrung der Eosinophilenzahl nach der Sonnenbestrahlung (nach der ersten Bestrahlung mittags 11 Uhr 139 mehr als am gleichen 'Tago um 8) (nach der zweiten Bestrahlung abends 6 Uhr $158 \mathrm{mehr}$ als am gleichen Tage um 8). Die Patientin wurde nach Wochen mit unverändertem Befunde entlassen. Das Körpergewicht hatte um $2 \mathrm{~kg}$ zugenommen, die Temperaturen waren nach wie vor subfebril und wurden durch die Bestrahlungen nicht in erkennbarer Weise verändert. Die Kranke hatto eine geringe Neigung zur Progredienz ihres Krankheitsprozesses und vortrug deshalb die Bestrahlungen. Freilich reagierte sie nicht so kräftig auf die Einzelbestrahlung, wio dio vorher geschilderte.

3. R. B., 22 Jahre alt, doppelseitige zur Schrumpfung neigende Spitzentuberkulose, Stadium I, Pleuraschwarte rechts, subfebrile Temperaturen, Bazillenbefund negativ, schlechter Ernährungszustand.

Absolute Eosinophilenzahlen

am 30. VI. 9 Uhr vormittags 307,6 Uhr abends 328.

am 1. VII. 8 Uhr vormittags 324 . 
Von 8.5 Uhr bis 8.20 Uhr Sonnenbestrahlung ohne Eintritt deutlicher Hautrötung. $11 \mathrm{Uhr}$ vormittags $319,2 \mathrm{Uhr}$ nachmittags $448,5 \mathrm{Uhr}$ nachmittags 421 ; am 2. VII. 8 Uhr vormittags 386 .

Von 8.10 Uhr bis 8.45 Uhr Sonnenbestrahlung mit Eintritt starker Hautrötung, Herzklopfen, Kopfschmerzen und allgemeiner nervöser Erregung

$12 \mathrm{Uhr}$ mittags 282, $3 \mathrm{Uhr}$ nachmittags 356, $6 \mathrm{Uhr}$ abends 294.

Hier findet sich bei der ersten Bestrahlung eine mäBige Vermehrung der Eosinophilenzahl (nachmittags 2 Uhr 124 mehr als morgens um 8). Bei der zweiten Bestrahlung aber am nächsten Tage, die 10 Minuten länger dauert, unter den Zeichen stärkerer Hautrötung und subjektiven Unbehagens eine Verminderung (mittags $12 \mathrm{Uhr} 104$ weniger als morgens um 8). Die Patientin war also bei dieser Bestrahlung überdosiert. Da das nicht wiederholt wurde, erholte sich die Kranke entsprechend ihrem geringen Befunde im Laufe von 2 Monaten glänzend und wurde bei ganz geringem physikalischen Befunde und einer Gewichtszunahme von $8,5 \mathrm{~kg}$ entlassen. Die subfebrilen Temperaturen verschwanden. Eine stärkere Temperaturreaktion trat auch nach der Überdosierung mit Sonne nicht ein.

\section{Künstliche Höhensonne.}

1. L. St., 28 Jahre alt, beginnende, linksseitige infiltrative Lungenspitzentuberkulose, Stadium I, kein Fieber, kein Auswurf, guter Ernährungszustand.

Absolute Eosinophilenzahlen

am 23. VIII. $11 \mathrm{Uhr}$ vormittags 317,5 Uhr nachmittags 328 ;

am 24. VIII. $8 \mathrm{Uhr}$ vormittags 227 , $11 \mathrm{Uhr}$ vormittags $315,5 \mathrm{Uhr}$ nachmittags 279 ;

am 25. VIII. $8 \mathrm{Uhr}$ vormittags 237 .

15 Minuten Höhensonne in der üblichen Weise, starke Hautrötung.

5 Uhr nachmittags 406 ;

am 26. VIII. 8 Uhr morgens 351,11 Uhr vormittags 302 , 5 Uhr nachmittags 398.

Hier findet sich bei der Höhensonnenbestrahlung eine beträchtliche Vermehrung der Eosinophilenzahl (nachmittags 5 Uhr $169 \mathrm{mehr}$ als morgens 8 Uhr). Der Fall lag prognostisch günstig und verlief dementsprechend.

2. M. S., 20 Jahre alt, teils infiltrative, teils schrumpfende Tuberkulose des rechten Oberlappens und der linken Spitze, Stadium II-III, Bazillenbefund positiv, subfebrile Temperaturen, sehr guter Ernährungszustand.

Absolute Zahl der Eosinophilen

am 19. VII. 8 Uhr vormittags 189, 12 Uhr mittags 217,6 Uhr abends 202 ;

am 20. VII. $8 \mathrm{Uhr}$ vormittags 192.

4 Minuten Höhensonne ohne wesentliche Rötung.

9 Uhr vormittags $189,12 \mathrm{Uhr}$ mittags $244,3 \mathrm{Uhr}$ nachmittags 291 , $6 \mathrm{Uhr}$ nachmittags 229 ;

am 29. VII. $8 \mathrm{Uhr}$ vormittags $210,12 \mathrm{Uhr}$ mittags $221,6 \mathrm{Uhr}$ abends 197;

am 30. VII. $8 \mathrm{Uhr}$ vormittags 187 .

7 Minuten Höhensonne deutliche Hautrötung.

$9 \mathrm{Uhr}$ vormittags $251,12 \mathrm{Uhr}$ mittags $232,3 \mathrm{Uhr}$ nachmittags 248 , $6 \mathrm{Uhr}$ abends 303 .

Hier findet sich bei der Höhensonnenbestrahlung beide Male eine deutliche Vermehrung der Eosinophilenzahlen (das erste Mal nach 4 Minuten Bestrahlung um 3 Uhr nachmittags 99 mehr als um 8 Uhr morgens) (das zweite Mal nach 7 Minuten Bestrahlung und eingetretener Hautrötung um 6 Uhr abends 116 mehr als um 8 Uhr morgens). Dic Kranke wurde nach längerer Behandlung wesentlich gebessert entlassen.

3. S. H., 17 Jahre alt, doppelseitige vorwiegend infiltrative Lungentuberkulose, Stadium II, Bazillenbefund positiv, subfebrile Temperaturen, starke Abmagerung.

Absolute Zahl der Eosinophilen

am 14. VIII. 8 Uhr vormittags 125,12 Uhr mittags 99, 6 Uhr abends 116 ;

am 15. VIII. $8 \mathrm{Uhr}$ vormittags 118 .

10 Minuten Höhensonne mäßige Hautrötung

11 Uhr vormittags 102,6 Uhr abends 85;

am 16. VIII. $8 \mathrm{Uhr}$ vormittags 117. 
Hier findet nach der Höhensonnenbestrahlung eine Verminderung der Eosinophilenzahlen statt. Der Kranke war von vornherein in einem unguten Zustande und wurde auch während der Behandlungszeit nicht gebessert.

\section{Therapeutische Röntgenbestrahlungen.}

1. K. K., 21 Jahre alt, doppelseitige vorwiegend indurierende Lungentuberkulose, Stadium II, Pleuraschwarte rechts, Bazillenbefund positiv, leicht subfebrile Temperaturen, mäßige Abmagerung.

Absolute Zahl der Eosinophilen

am 22. VI. $8 \mathrm{Uhr}$ vormittags 310 ;

am 23. VI. 8 Uhr vormittags 330,12 Uhr mittags 400,6 Uhr abends 350 ;

am 24. VI. $8 \mathrm{Uhr}$ vormittags 325 .

Röntgenbestrahlung nachmittags 5 Uhr links hinten oben $6 \mathrm{mal} 8 \mathrm{~cm}$, Filter: 0,5 Zink plus 1,0 Aluminium, $23 \mathrm{~cm} \mathrm{F.} \mathrm{H.,} 15$ Minuten Dauer, Instrumentarium Reiniger Gebbert und Schall (Symmetrie).

6 Uhr abends 250;

am 25. VI. 9 Uhr vormittags 150,12 Uhr mittags 250,6 Uhr abends 320 ;

am 27. VI. 8 Uhr morgens 320 .

Röntgenbestrahlung rechts hinten oben genau wie am 24. links.

9 Uhr morgens 187, 4 Uhr nachmittags 337,7 Uhr abends 220;

am 28. VI. 8 Uhr morgens 263,4 Uhr nachmittags 217;

am 30. VI. $8 \mathrm{Uhr}$ vormittags 250 .

Röntgenbestrahlung des rechten Hjlus sonst wie am 24. und 27.

9 Uhr vormittags 297,4 Uhr nachmittags 290 ;

am 1. VII. 11 Uhr vormittags $260,4 \mathrm{Uhr}$ nachmittags 155 ;

am 2. VII. 10 Uhr vormittags 206.

Hier findet nach den Röntgenbestrahlungen eine deutliche Verminderung der Eosinophilenzahlen statt. Nur am 30. findet sich eine geringè Vermehrung. Das subjektive Befinden des Kranken war zunächst ein gutes, auch der objektive Lungenbefund besserto sich zunächst insofern als die Rasselgeräusche geringer wurden, das Körpergewicht nahm um $1 \mathrm{~kg} \mathrm{zu}$. Die Temperatur blieb subfebril, der Bazillenbefund positiv. Im Laufe der Zeit aber nahm der Kranke wieder ab und wurde schließlich in schlechterem Zustand entlassen, als er gekommen war.

2. M. A., 55 Jahre alt. Ausgedehnte stark schrumpfende Tuberkulose beider Lungen, Stadium II, Bazillenbefund positiv, kein Fieber, mäßige Abmagerung.

Absolute Eosinophilenzahl

am 19. VI. 8 Uhr vormittags 365,12 Uhr mittags 388,6 Uhr nachmittags 363 ; am 20 . VI. 7 Uhr vormittags 378 .

Röntgenbestrahlung links hinten oben 6 mal $8 \mathrm{~cm}$, Filter: 0,5 Zink plus 1,0 Aluminium, $23 \mathrm{~cm}$ F. H. 15 Minuten Dauer, Instrumentarium wie bei Fall 1.

am 22. VI.

$8 \mathrm{Uhr}$ vormittags $363,3 \mathrm{Uhr}$ nachmittags 384, 6 Uhr abends 505;

Morgens 8 Uhr Röntgenbestrahlung des linken Hilus wie am 20. VI.

$9 \mathrm{Uhr}$ vormittags $566,3 \mathrm{Uhr}$ nachmittags 540 ;

am 24. VI. $7 \mathrm{Uhr}$ vormittags 546.

Röntgenbestrahlung rechts hinten oben, wie am 20. VI.

9 Uhr vormittags 594,3 Uhr nachmittags 540,6 Uhr abends 600 ;

am 25. VI. 9 Uhr vormittags 688,3 Uhr nachmittags 664 ;

am 27. VI. 7 Uhr vormittags 558 .

Röntgenbestrahlung des rechten Hilus wie am 20. VI.

9 Uhr vormittags 434,7 Uhr abends 250 .

An diesem Tage starker Röntgenkater!

am 28. VI. 9 Uhr vormittags 494,3 Uhr nachmittags 468 ;

am 30. VI. 
Morgens 7 Uhr Röntgenbestrahlung rechts vorn oben, wie am 20. VI. aber nur 10 Minuten.

9 Uhr vormittags 428,3 Uhr nachmittags 448,6 Uhr abends 454;

am I. VII. 8 Uhr vormittags 403 ;

am 2. VII. $7 \mathrm{Uhr}$ vormittags 440 .

Röntgenbestrahlung links vorn oben, wie am 20. VI. aber nur 10 Minuten.

9 Uhr vormittags 454,3 Uhr nachmittags 526,6 Uhr abends 5ll;

am 5. VII. 9 Uhr vormittags 434 .

Hier findet nach den Röntgenbestrahlungen zunächst eine Vermehrung der Eosinophilen statt (am 20. abends um 6 Uhr 127 mehr als morgens um 7 Uhr) während) des ganzen 22. durchschnittlich 150 mehr als vor den Bestrahlungen) (am 24. und 25. nach der dritten Bestrahlung Zahlen von durchschnittlich etwa 640 gegenüber durchschnittlich etwa 375 vor den Bestrahlungen). Dann erfolgt am 27. auf eine Hilusbestrahlung ein starker Röntgenkater, bei dem die Zahlen weit bis unter den Anfangswert stürzen (von durchschnittlich 375 vor den Bestrahlungen auf $250 \mathrm{~d}$. h. also um 175 gegenüber dem Durchschnitt und 208 gegenüber dem Morgenwert vor der Bestrahlung. Bei späteren kürzer angesetzten Bestrahlungen heben sich die Zahlen wieder etwas, übertreffen auch noch die Anfangswerte, erreichen aber nie wieder die Höhe, wie im besten Zeitpunkte der Kur vor dem Kater. Das subjektive Befinden des Kranken war abgesehen von dem Kater gut, er ließ sich gern bəstrahlen. Objektiv nahm das Gewicht langsam ab, der Lungenbefund wurde allmählich schlechter. Fieber trat nie auf.

3. C. H. 37 Jahre alt, doppelseitige vorwiegend fibröse Tuberkulose, Stadium I-II, Bazillenbefund negativ, Temperatur normal, guter Ernährungszustand.

Absolute Zahl der Eosinophilen

am 23. VI. 8 Uhr vormittags 150,12 Uhr mittags 176, 6 Uhr abends 178 ;

am 24. VI. 8 Uhr vormittags 138.

Röntgenbestrahlung rechts hinten oben $6 \mathrm{mal} 8 \mathrm{~cm}$, Filter: 0,5 Zink plus 1,0 Aluminium, $23 \mathrm{~cm}$ F. H., 15 Minuten Dauer. Instrumentarium Reiniger, Gebbert und Schall (Symmetrie).

10 Uhr vormittags 120,6 Uhr abends 150 ;

am 25. VI. 12 Uhr mittags 110,6 Uhr abends 150 ;

am 27. VI. 8 Uhr vormittags 135 .

Röntgenbestrahlung links hinten oben sonst wie am 24. VI.

9 Uhr vormittags $187,4 \mathrm{Uhr}$ nachmittags $170,7 \mathrm{Uhr}$ abends 173;

an 28. VI. 8 Uhr vormittags 174,4 Uhr nachmittags 175 ;

am 30. VI. 8 Uhr vormittags 184 .

Röntgenbestrahlung des rechten Hilus wie am 24. VI.

11 Uhr vormittags 175,4 Uhr nachmittags 155 ;

am 1. VII. $10 \mathrm{Uhr}$ vormittags $130,4 \mathrm{Uhr}$ nachmittags 150 ;

am 2. VII. 10 Uhr vormittags 170 ;

am 3. VII. 8 Uhr vormittags 157 .

Röntgenbestrahlung rechts vorne oben wie sonst am 24. VI.

10 Uhr vormittags 132, 5 Uhr nachmittags $152,7 \mathrm{Uhr}$ abends 158;

am 4. VII. $9 \mathrm{Uhr}$ vormittags $148,4 \mathrm{Uhr}$ nachmittags 152.

Hier wird durch die Röntgenbestrahlungen die Eosinophilenzahl nur ganz unwesentlich, meist im Sinne einer Verminderung beeinflußt. Nur am 27. nach der zweiten Bestrahlung findet in der genannten Zeit eine Vermehrung um 52 statt. Das subjektive Befinden des Kranken war gut, es trat im Laufe der Beobachtung eine Gewichtszunahme um 2,5 kg auf, aber der objektive Befund war bei der Entlassung nach Wochen eher schlechter als zu Beginn.

Durch die vorstehenden Beispiele soll in erster Linie demonstriert werden, daß eine Beeinflussung der Eosinophilenkurve durch Maßnahmon der physikalischen Therapie möglich ist. Diese Beispiele stellen nur sehr teilweise ordnungsmäßige Behandlungsserien dar, beschränken sich vielmehr zum größeren Teile auf Stichproben, wie sie bei Kranken gemacht werden konnten, die angesichts des langwierigen Verlaufes ihres Leidens noch dieses oder jenes Hilfs- 
mittel angewandt wissen wollten. Sie zeigen, daß die gebräuchlichen physikalisch-therapeutischen Maßnahmen imstande sind, die Funktionskurve des Knochenmarkes sowohl zum Steigen, wie auch zum Fallen zu bringen. Naturgemäß darf man dabei auf kleine Zahlen als solche kein entscheidendes Gewicht legen. Schon die normalen Werte schwanken andauernd. Nur die'Tatsache, daß die Kurve stark steigt, oder undeutlich beeinflußtwird, oder stark sinkt, ist von Bedeutung.

Im allgemeinen gewinnt man auch hier wieder den Eindruck, daß immer wiederholtes kräftiges Steigen der Funktionskurve bei solchen Kranken vorkommt, welche die Therapie gut vertragen, während undeutliche Beeinflussung oder Sinken der Funktionskurve bei Kranken beobachtet wird, deren Zustand sich allmählich oder krisenhaft verschlechtert. Das tritt sowohl bei den progredienten Fällen auf, bei denen die Reaktionsfähigkeit sowieso sinkt und jeder ernstliche therapeutische Eingriff dieses Sinken eher noch befördert, als auch bei den günstiger gelagerten Prozessen, bei denen nur momentan zu rasch vorgegangen ist. U̇berdosierung mit folgendem krisenhaften Unbehagen nach einzelnen Licht- und Röntgendosen.

Die Eosinophilen-Untersuchung bestätigt somit das, was jeder erfahrene Phthiseotherapeut weiß, nämlich, daß man mit Anwendung natürlicher und künstlicher Sonne bei aktiven Lungenprozessen überaus vorsichtig sein muß. Bezüglich der Röntgenbestrahlung zeigt sie, daß auch bei wenig ausgedehnten, indurierenden Prozessen Zurückhaltung am Platze ist. Im großen und ganzen gewannen wir den gleichen Eindruck, wie beim Tuberkulin: Fälle, die günstig verlaufen, lassen schon frühzeitig eine Steigerung der Eosinophilenkurve erkennen. Bei allen Fällen, die zu ungünstigem Verlauf neigen, ist das Umgekehrte der Fall. Darum möchten wir auch hier den Satz umkehren und sagen: Wenn die Eosinophilen während der therapeutischen Beeinflussung eine rasche und kräftige Tendenz zum Steigen haben, dann darf man beruhigt sein und vorsichtig tastend allmählich kräftiger zufassen. Erfahren sie keine deutliche Beeinflussung, so soll man mit den therapeutischen Dosen zunächst nicht steigen. Sinken sie stark, so soll man die Kur alsbald unterbrechen. Dabei ist natürlich wiederum aus einem einzelnen Versager noch kein für längere Zeit sicher bindender Schluß zu machen. Ein solcher kann durch eine Utberdosierung crklïrt werden, die zwar nicht nach dem allgemeinen Schema, aber doch nach der besonderen Lage des Falles erklärt ist. Besonders werden interkurrente Indispositionen hier eine Rolle spielen. Auch kann die gleiche Krankheit heute in einer ungünstigeren und weniger reaktiven Phase sich befinden, wie in einigen Wochen oder Monaten. Das, was wir durch die Eosinophilenprüfung erfahren, ist und bleibt immer nur das augenblickliche Verhältnis zwischen Belastung und Reaktionsfähigkeit. Erst mehrere solcher Stichproben lassen sich zu einem längeren Faden ausspinnen.

Wir fassen zusammen: 1. Es ist allgemein anerkannt, daß ein Ansteigen der Eosinophilenkurve nach Tuberkulindarreichung als ein prognostiseh gïnstiges Zeichen angesehen werden darf.

2. Die Erkenntnis, daß ein Gleichbleiben oder gar Sinken ker Kurve $14 \mathrm{~m}$ gekehrt gewertet werden darf, bricht sich immer mehr Bahı. 
3. Es besteht bezüglich der Eosinophilenkurve und der daraus zu ziehenden Schlüsse kein prinzipieller Unterschied zwischen den verschiedenen Tuberkulinen und den üblichen Vakzinen.

4. Die physikalisch-therapeutischen Maßnahmen wirken in ähnlichem Sinne auf die Eosinophilenkurve ein, wie die vorstehend genannten chemischen Mittel.

5. Dementsprechend sehen wir das Verhalten der Eosinophilen als einen allgemeinen Maßstab dor Reaktionsfähigkeit des Organismus im Kampfe gegen Infektionen an.

\section{Literatur.}

1. Brösamlen, Dtsch. Arch. f. klin. Med. 115. 146.

2. Fauconnet, Dtsch. Arch. f. klin. Med. 82.

3. Grawitz, Zeitschr. f. klin. Med. 22.

4. Botkin, Dtsch. med. Wochenschr. 1892.

5. Brösamlen, Dtsch. Arch. f. klin. Med. 118. 163.

6. Schenitzki, Zeitschr. f. exp. Pathol. u. Therap. 19. 228.

7. Brösamlen, Zeitschr. f. exp. Pathol, u. Therap. 20. H. 3.

8. Schlecht, Arch. f. exp. Pathol. u. Pharmakol. 67. 192.

9. Sehlecht und Schwenker, Dtsoh. Arch. f. klin. Med. 108.

10. Hayek, Immunbiologie, Dispositions- und Konstitutionsforschung. Berlin. Julius Springer 1921.

11. Zi mmer, Münch. med. Wochenschr. 191. Nr. 18. 592.

12. Fränkel, Fortschr. a. d. Geb. d. Röntgenstr. 26. 43.

13. Blumenfeld, Zeitschr. f. exp. Pathol. u. Therap. 20. 14.

14. Romberg, Zeitschr. f. Tuberkul. 34. 191.

15. Rolly, Münch. med. Wochenschr. 68. 835 .

16. Dunger, Münch. med. Wochenschr. 1910. Nr. 37.

17. Brösamlen, Zeitschr. f. exp. Pathol. u. Therap. 20. H. 1. 